\title{
Random motions at the velocity of light and relativistic quantum mechanics
}

\author{
Nicola Cufaro Petroni $†$ and Jean Pierre Vigier $\ddagger$ \\ $\nmid$ Istituto Nazionale di Fisica Nucleare, Sez. di Bari, Italia, and Istituto di Fisica dell' \\ Università Bari, Italia \\ $\ddagger$ Equipe de recherche associée au CNRS no 533, Institut $\mathrm{H}$ Poincaré, 11 , rue $\mathrm{P}$ et $\mathrm{M}$ \\ Curie, 75231 Paris Cedex 05, France
}

Received 22 September 1982, in final form 26 September 1983

\begin{abstract}
With the physical hypothesis of the existence of a covariant subquantum vacuum, Dirac's aether, we show that: (i) the idea that subquantum random jumps occur at the velocity of light is a consequence of the introduction of stochastic fluctuations into the $g_{\mu \nu}$ field of general relativity and (ii) the Klein-Gordon equation can be deduced, in a new simple way, from a stochastic process on the set of the four possible space-time directions of the velocity of light.
\end{abstract}

\section{Introduction}

The main new points in recent developments of the stochastic interpretation of quantum mechanics are the following.

(a) The de Broglie waves are just collective motions on the top of a real physical covariant aether (Dirac 1951) which surrounds oscillator-like particles, like sound waves around planes flying at Mach 1. Following de Broglie these waves 'pilot' the particles along average drift lines of flow associated with the effects of a stochastic quantum potential.

(b) The introduction of such a real physical aether implies a non-local quantum potential which presents the remarkable property that the corresponding action-at-adistance is perfectly causal in the Einstein sense of the term (Cufaro Petroni and Vigier 1979b). This point is important because a series of experiments (Freedman and Clauser 1972, Holt and Pipkin 1974, Faraci et al 1974, Kasday et al 1975, Clauser 1976, Fry and Thompson 1976, Wilson et al 1976 and Bruno et al 1977), culminating in the recent one of Aspect et al $(1981,1982)$, suggests that the non-separability of correlated systems deduced from the quantum formalism is a real fact and that Bell's inequality (Bell 1964) is badly violated. In this case the problem raised by the Einstein-PodolskyRosen paradox (1935) will have a solution in the sense that the quantum mechanical implication of space-like correlations between two linear polarisers which measure the rate of coincidence between the relative orientations of pairs of photons emitted in the S state will be experimentally verified. If the forthcoming crucial experiments proposed by Aspect $(1975,1976)$ and Rapisarda (Falciglia et al 1979, Garuccio et al 1981, Garuccio and Rapisarda 1981) confirm these conclusions, the only possible causal way out of the resulting contradiction between relativity and the quantum theory of measurement seems to lie, as suggested in recent papers (Cufaro Petroni et al 1981), 
in the direction of the utilisation of the stochastic interpretation of quantum mechanics in order to introduce a suitable relativistic action-at-a-distance.

(c) The wave elements and the particles jump at random from one drift line of flow to another at the velocity of light. This idea is essential in this model since Lehr and Park (1977), Guerra and Ruggiero (1978), Vigier $(1979,1980)$ and Cufaro Petroni and Vigier (1979a, 1981, 1982, 1983) have deduced therefrom (i) the relativistic generalisation of Nelson's equation (Nelson 1966), (ii) the exact form of the relativistic wave equations, (iii) the numerical value of the corresponding diffusion coefficient $D=\hbar / 2 m$.

The aim of the present paper is to develop this stochastic model, firstly introduced by Bohm $(1952,1953)$ and Bohm and Vigier (1954, 1958), one step further, i.e.

(1) To show in $\$ 2$ that the basic idea that subquantum random jumps occur at the velocity of light is a natural consequence of introducing stochastic fluctuations into the $g_{\mu \nu}$ field of general relativity.

(2) To expose in the subsequent sections a new method for deducing the relativistic quantum equations for spinless particles from a Markov process which is explicitly built by a random walk on a space-time lattice with the only physical assumption that the stochastic jumps occur at the velocity of light. This approach, because of the evidence of its construction, will allow a new very clear insight into the chaotic processes subjacent to the quantum behaviour. More precisely: in $\S 3$ we construct a twodimensional space-time lattice and define our random walks with a statistical weighting for each possible trajectory of a single spinless particle; in $\S 4$ we finally deduce, from this Markov process, the Klein-Gordon equation.

\section{Einstein's subquantum random aether model}

The idea of using random fluctuations of the $g_{\mu \nu}$ field (considered by Einstein himself (1924) to be a possible representation of a real all pervading material field) as the origin of the real quantum forces which justify the stochastic interpretation of quantum mechanics is not new in the literature. Indeed Einstein's ideas on the question were reactivated by Janossy (1972) who considered the cosmological term $\Lambda g_{\mu \nu}$ in

$$
R_{\mu \nu}-\frac{1}{2} g_{\mu \nu} R+\Lambda g_{\mu \nu}=\chi T_{\mu \nu}
$$

as a representation of the energy momentum tensor distribution of the background gravitational aether. Moreover, the introduction of a random $g_{\mu \nu}$ component (which goes back to March 1934, 1937, Markov 1958, Yukawa 1966 and Blokhintsev 1974) has been recently revived by Frederick (1976), Namsrai (1980) and Cerofolini (1980) by the following conditions.

(a) At each world point the tangent space-time is not defined by the Minkowsky $\delta_{\mu \nu}$ but by $F^{2} \delta_{\mu \nu}+\delta \tilde{g}_{\mu \nu}$ where denotes stochastic tensor components and $F$ a real Weyl gauge function.

(b) All measurements of dynamical variables correspond to contravariant components of tensors.

(c) The probability $P(r, t)$ of finding a particle can be written $P(r, t)=A(r, t) \sqrt{-g}$ where $g$ is the determinant of the metric.

(d) The $\delta \tilde{g}_{\mu \nu}$ are small and practically add linearly; for example the metric due to different physical situations 1 and 2 is $g_{\mu \nu}(3)=\frac{1}{2}\left[g_{\mu \nu}(1)+g_{\mu \nu}(2)\right]$. 
Assumptions (a), (b), (c), (d) imply the following consequences.

(1) If one denotes the metric at the point 1 by $\tilde{g}_{\mu \nu}(1)$ we cannot determine it precisely but only ask for $P\left(\tilde{g}_{\mu \nu}\right)$ which is the probability of a particular $\tilde{g}_{\mu \nu}$. However, we can define its average value $\dot{g}_{\mu \nu}=\left\langle\tilde{g}_{\mu \nu}\right\rangle$ over a four-dimensional volume element, so that $\tilde{g}_{\mu \nu}=\left\langle\tilde{g}_{\mu \nu}\right\rangle$ with $\left\langle\delta \tilde{g}_{\mu \nu}\right\rangle=\left\langle\partial^{\alpha} g_{\mu \nu}\right\rangle=0$. The fluctuating $\delta \tilde{g}_{\mu \nu}$ field can thus be compared to a chaotic superposition of random wavelets on a curved regular sea. This is physically reasonable since gravitational waves originating from all independent (practically uncorrelated) sources in the universe are expected to build a random background gravitational noise i.e. the 'gravitational vacuum'. Moreover, at each point one can write $g_{\mu \nu}=\dot{g}_{\mu \nu}+\delta \tilde{g}_{\mu \nu}=\dot{d}^{2} \delta_{\mu \nu}+\delta \tilde{g}_{\mu \nu}$ where $\dot{d}$ represents the average value of the local dilatation $d$ which contains an average plus a random part: the metric $\dot{d}^{2} \delta_{\mu \nu}$ being locally conformal to the initial undisturbed metric.

(2) The use of contravariant quantities in measurement theory is necessary if we represent test particles as massive singularities of the $g_{\mu \nu}$ field which thus necessarily follow geodetics. Indeed the random $\tilde{g}_{\mu \nu}$ imply fluctuating geodetics (i.e. random gravitational forces) since we have on each such particle the Euler-Lagrange equations

$$
\ddot{x}^{\mu}+\left\{\begin{array}{c}
\bar{\mu} \\
\nu
\end{array}\right\} \dot{x}^{\nu} \dot{x}^{\alpha}=0
$$

where $\{\sim$ are now fluctuating Christoffel symbols and denotes the proper time derivative. Relation (2.2) evidently generates a random set of motions around the usual mean geodetics defined from $\stackrel{g}{g}_{\mu \nu}$. These motions, which can be neglected for macroscopic bodies, imply an irreducible stochastic character upon all motions of microscopic test particles: an evident possible basis for the stochastic interpretation of quantum mechanics. Since we must introduce measurable $\mathrm{d} x^{\mu}$ in (2.2) we must be able to define the distance to our singular test particles. Calling this distance $\bar{r}\left(\equiv \bar{\xi}^{i}\right)$ the covariant equivalent of the radial coordinate $r$ is $\xi_{i}$ and $\xi_{i}=g_{1 \nu} \xi^{\nu}=g_{11} \xi^{1}=$ $r(1-2 \mathrm{Gm} / r)^{-1}$ which yields for the contravariant distance $\int_{0}^{r} \mathrm{~d} r=\bar{r}$ in contradistinction with the covariant distance $\bar{\xi}_{1}=\int_{0}^{\bar{r}} \mathrm{~d} r(1-2 \mathrm{Gm} / \mathrm{r})^{-1}=\infty$ so that only the contravariant distance is observable.

(3) The stochastic random motions induced by the $\tilde{g}_{\mu \nu}$ occur at the velocity of light. This is the essential property of our model. It can be demonstrated as follows. Following Frederick's (1976) line of argument we start with a particle at rest (for an inertial observer) at a space point $\theta_{0}$. After a time $\mathrm{d} t$ the Euler-Lagrange equations (2.2) yield a probability $D_{1}(x)$ of being in a region bounded by $x$ and $x+\mathrm{d} x$. After a second time interval $\mathrm{d} t$ the new distribution is $D_{1+2}(x)$. From probability theory (Cramer 1955) this is the convolution $D_{1+2}(x)=\int_{-\infty}^{\infty} D_{1}(y) D_{2}(x-y) \mathrm{d} y$. Assuming vacuum isotropy (so that the Euler-Lagrange equations give the same distribution independently of the starting point) we have for our Brownian-like motion $D_{1}(x)=$ $D_{2}(x)$. Since $g_{\mu \nu}(x) \equiv\left\{g_{\mu \nu}\left(x_{1}\right), g_{\mu \nu}\left(x_{2}\right) \ldots\right\}$ and thus $D_{i}(x) \equiv\left\{D_{1}(x), D_{2}(x) \ldots\right\}$ are identically distributed random variables, the motion of any test particle is equal to the repeated convolution $D_{1+2+\ldots}(x) \ldots$ which yields a normal distribution by the central limit theorem. The position spread of the particle at $t>0$ is a gaussian. To calculate the spreading velocity we remark that after $N$ such convolutions ( $N$ large) we obtain a normal distribution with variance $\sigma^{2}$. Still according to the central limit theorem (Cramer 1955), this distribution is $N$ times the variance of $D_{1}(x)$. If we represent this variance of $D_{1}(x)$ by $a^{2}$, i.e.

$$
\left(\operatorname{var}\left(D_{1}\right)\right)^{1 / 2}=a
$$


we see that the distribution $D_{1}$ is obtained after $\mathrm{d} t$ so that we can write after $N$ convolutions

$$
\Delta x=\left[\operatorname{var}\left(D_{1+2+\ldots+N}\right)\right]^{1 / 2}=N a
$$

resulting from $N$ time intervals $\mathrm{d} t$. This yields

$$
\Delta x / \Delta t=N a / N=a
$$

so that the initially localised test particle spreads with a constant velocity $a$ which must be equal to $c$ since the result is frame independent. The same result is obtained with a particle with a definite position and a momentum distribution with the difference of having a different distribution $D_{1}$ due to the uncertainty of the particle's direction of propagation.

\section{Random walk at the velocity of light}

Starting from the preceding result we shall now attempt, following a very simple classical analysis (Avez 1976), to analyse stochastic random walks at the velocity of light. To simplify our demonstration we will limit ourselves to the case in which we assume that this random walk occurs on a square lattice in a two-dimensional space-time (see figure 1).

We remark at this stage that, properly speaking, the Markov process describing the space-time trajectory of our particle takes place on the velocity space which, if the particle always jumps at the velocity of light, reduces, as we will soon see, to the set of the four possible space-time directions of the velocity of light. To analyse our random walk at the velocity of light we will describe our two-dimensional space-time with the coordinates $x^{0}, x^{1}$, and we will make a limit process where in each step we will suppose that our particle, starting from an arbitrary point $P\left(x^{0}, x^{1}\right)$, can make only jumps of fixed length and always at the velocity of light. As is shown in figure

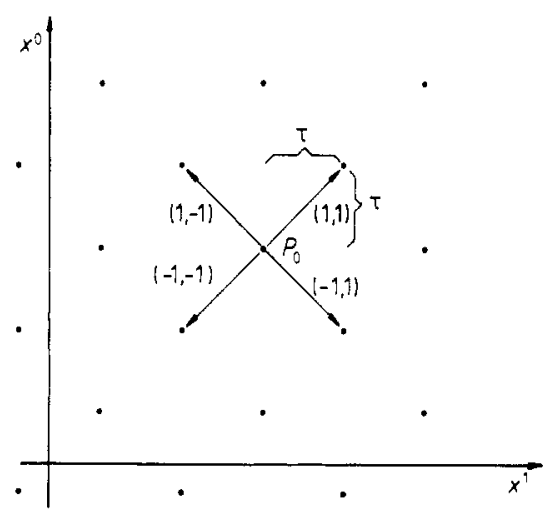

Figure 1. Space-time lattice of dimension $\tau$ and starting point $P_{0}$. For each possible direction of the first jump we marked the corresponding value of the couple $(t, s)$.

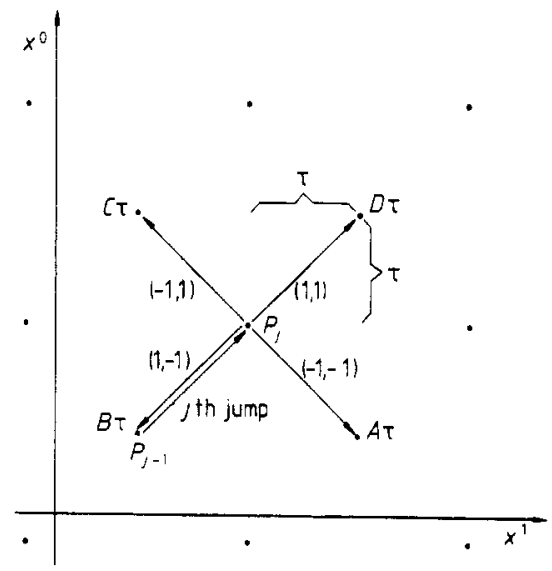

Figure 2. An example of the four possible successions of two jumps. For each possible $(j+1)$ th jump we marked the value of the couple $\left(\varepsilon_{j}, \eta_{j}\right)$ and the corresponding probability. 
1 , this prescription completely determines the lattice of all the possible positions of the particle. On this lattice the particle can follow an infinity of possible trajectories.

In our calculation we will consider first a lattice with fixed dimensions: indeed for each jump we pose

$$
\Delta x^{0}=t \tau, \quad \Delta x^{1}=s \tau \quad(t, s= \pm 1)
$$

so that for the velocity we always have (we choose here $\hbar=c=1$ )

$$
v=\Delta x^{1} / \Delta x^{0}=s / t= \pm 1 \text {. }
$$

Here $\tau$ is the parameter which fixes the lattice dimensions: of course, in order to recover the quantum equations, we will consider later the limit $\tau \rightarrow 0$. Moreover, it is clear from (3.1) and figure 1 that on this lattice we also consider the possibility of trajectories running backward in time: we will interpret them as trajectories of antiparticles running forward in time following the usual Feynman (1949) interpretation.

In order to describe random walks on this lattice we consider the following Markov process on the set of the four possible directions of the velocity: we define two sets of stochastic variables $\left\{\varepsilon_{j}\right\},\left\{\eta_{j}\right\}$, with $j \in N$, in such a way that the only possible values of each $\varepsilon_{j}$ and $\eta_{j}$ are \pm 1 following this prescription

$\varepsilon_{j}=\left\{\begin{array}{r}1 \\ -1\end{array}\right.$ if in the $(j+1)$ th jump the sign of the velocity $\left\{\begin{array}{c}\text { doesn't change } \\ \text { changes }\end{array}\right.$

$\eta_{j}=\left\{\begin{array}{r}1 \\ -1\end{array}\right.$ if in the $(j+1)$ th jump the direction of the time $\left\{\begin{array}{c}\text { doesn't change } \\ \text { changes }\end{array}\right.$

with respect to the preceding $j$ th jump. It means that the realisation of the signs of $\varepsilon_{j}, \eta$, determines one of the four possible directions of the $(j+1)$ th jump on the ground of the direction of the $j$ th jump, as we can see in figure 2 .

Of course a sequence $\left\{\varepsilon_{j}, \eta_{j}\right\}$, with $j \in N$, of values of these stochastic variables completely determines one of the infinite possible trajectories except for the first jump because there is no 'preceding' jump for it. Thus, starting from $P_{0}\left(x^{0}, x^{1}\right)$, in the first jump we can get one of the four possible points $P_{1}\left(x^{0}+t \tau, x^{1}+s \tau\right)$ and after $N$ jumps, as we can easily see by direct calculation, one of the points $P_{N}\left(x^{0}+t T_{N}, x^{1}+s D_{N}\right)$ where

$$
\begin{aligned}
& T_{N}=\tau\left(1+\eta_{1}+\eta_{1} \eta_{2}+\ldots+\eta_{1} \eta_{2} \ldots \eta_{N-1}\right) \\
& D_{N}=\tau\left(1+\varepsilon_{1} \eta_{1}+\varepsilon_{1} \varepsilon_{2} \eta_{1} \eta_{2}+\ldots+\varepsilon_{1} \varepsilon_{2} \ldots \varepsilon_{N-1} \eta_{1} \eta_{2} \ldots \eta_{N-1}\right) .
\end{aligned}
$$

We come now to the problem of the assignment of a statistical weight to each trajectory. In order to do that we introduce for each $(j+1)$ th jump a probability for each realisation of the signs of the corresponding $j$ th couple $\varepsilon_{j}, \eta_{j}$. In table 1 we have listed these probabilities for a general $\varepsilon_{j}, \eta_{j}$ couple, namely for each possibility of each

Table 1. Probabilities for the four possible successions of two jumps.

\begin{tabular}{rrl}
\hline$\varepsilon_{j}$ & $\eta_{j}$ & Probability \\
\hline-1 & -1 & $A \tau$ \\
1 & -1 & $B \tau$ \\
-1 & 1 & $C \tau$ \\
1 & 1 & $D \tau$ \\
\hline
\end{tabular}


step of the Markov process on the velocity space. Moreover, we suppose that $A, B$, $C, D$ are constant and positive over all the space-time.

Among these four constants we can also pose a relation that can be justified as a principle of mass fiux conservation. If we consider for example, as in figure 2, a particle arriving at $P_{j}$ in its $j$ th jump, we must remember, for the $(j+1)$ th jump, that the particles running backward in time must be considered like antiparticles going forward in time. In this perspective if we want to conserve the flux of particles across the point $P_{j}$ between the $j$ th and the $(j+1)$ th jumps, we must remark that:

(a) in the $j$ th jump we have only a particle going right;

(b) in the $(j+1)$ th jump we have a 'fraction' $D \tau$ of the particle and $B \tau$ of the antiparticle going right and a 'fraction' $C \tau$ of the particle and $A \tau$ of the antiparticle going left.

If we consider that particles and antiparticles have the same mass, the mass flux conservation across $P_{j}$ gives finally

$$
(-A+B-C+D) \tau=1 \text {. }
$$

This relation will be very useful in the subsequent derivation.

\section{Relativistic wave equation}

In order to derive the Klein-Gordon equation we consider a function $f\left(x^{0}, x^{1}\right)$ defined over all the space-time and, generally speaking, with complex values, and then we define the following set of functions

$$
F_{N}^{t, s}\left(x^{0}, x^{1}\right)=\left\langle f\left(P_{N}\right)\right\rangle=\left\langle f\left(x^{0}+t T_{N}, x^{1}+s D_{N}\right)\right\rangle .
$$

Here $\langle\cdot\rangle$ indicates an average made over all the possible points $P_{N}$ attained following trajectories constituted by $N$ jumps starting from $P_{0}\left(x^{0}, x^{1}\right)$ with a first jump made in the direction fixed by $(t, s)$.

In fact it is clear that the terminal point $P_{N}$ is not uniquely determined by the initial point $P_{0}$ and the number of jumps $N$ because of the possibility of choosing different trajectories of $N$ jumps. Of course in the average, the statistical weight of each $P_{N}$ is calculated from the probabilities associated to the trajectories which lead to $P_{N}$ as stated in the previous section. We remark finally that, because of the arbitrariness of the starting point $P_{0}$, the function $F_{N}^{t, s}$ is defined over all the space-time.

We can start to make this average from the first jump so that, remembering (4.1) and (3.3):

$$
\begin{aligned}
F_{N}^{t, s}\left(x^{0}, x^{1}\right)= & \left\langlef \left[ x^{0}+t \tau+t \tau \eta_{1}\left(1+\eta_{2}+\ldots+\eta_{2} \ldots \eta_{N-1}\right), x^{1}+s \tau+s \tau \varepsilon_{1} \eta_{1}\right.\right. \\
& \left.\left.\times\left(1+\varepsilon_{2} \eta_{2}+\ldots+\varepsilon_{2} \ldots \varepsilon_{N-1} \eta_{2} \ldots \eta_{N-1}\right)\right]\right\rangle \\
= & \left\langle f\left(x^{0}+t \tau+t \eta_{1} T_{N-1}, x^{1}+s \tau+s \varepsilon_{1} \eta_{1} D_{N-1}\right)\right\rangle \\
= & D \tau F_{N-1}^{t, s}\left(x^{0}+t \tau, x^{1}+s \tau\right)+A \tau F_{N-1}^{-t, s}\left(x^{0}+t \tau, x^{1}+s \tau\right) \\
& +B \tau F_{N-1}^{-t,-s}\left(x^{0}+t \tau, x^{1}+s \tau\right)+C \tau F_{N-1}^{t,-s}\left(x^{0}+t \tau, x^{1}+s \tau\right)
\end{aligned}
$$

and using (3.4), that is $D \tau=1+(A-B+C) \tau$, we get

$$
\begin{aligned}
F_{N}^{t, s}\left(x^{0}, x^{1}\right)= & F_{N-1}^{t, s}\left(x^{0}+t \tau, x^{1}+s \tau\right) \\
& +A \tau\left[F_{N-1}^{-t, s}\left(x^{0}+t \tau, x^{1}+s \tau\right)+F_{N-1}^{t, s}\left(x^{0}+t \tau, x^{1}+s \tau\right)\right]
\end{aligned}
$$




$$
\begin{aligned}
& +B \tau\left[F_{N-1}^{-t,-s}\left(x^{0}+t \tau, x^{1}+s \tau\right)-F_{N-1}^{t, s}\left(x^{0}+t \tau, x^{1}+s \tau\right)\right] \\
& +C \tau\left[F_{N-1}^{t,-s}\left(x^{0}+t \tau, x^{1}+s \tau\right)+F_{N-1}^{t, s}\left(x^{0}+t \tau, x^{1}+s \tau\right)\right]
\end{aligned}
$$

We pass now to the limit $N \rightarrow \infty$ (and $\tau$ fixed): if we indicate with $F^{\text {t,s }}$ the functions for $N \rightarrow \infty$ we have from (4.3)

$$
\begin{aligned}
F^{t, s}\left(x^{0}, x^{1}\right)= & F^{t, s}\left(x^{0}+t \tau, x^{1}+s \tau\right)+A \tau\left[F^{-t, s}\left(x^{0}+t \tau, x^{1}+s \tau\right)+F^{t, s}\left(x^{0}+t \tau, x^{1}+s \tau\right)\right] \\
& +B \tau\left[F^{-t,-s}\left(x^{0}+t \tau, x^{1}+s \tau\right)-F^{t, s}\left(x^{0}+t \tau, x^{1}+s \tau\right)\right] \\
& +C \tau\left[F^{t,-s}\left(x^{0}+t \tau, x^{1}+s \tau\right)+F^{t, s}\left(x^{0}+t \tau, x^{1}+s \tau\right)\right]
\end{aligned}
$$

and then

$$
\begin{aligned}
-\frac{F^{t, s}\left(x^{0}+t \tau,\right.}{\left.x^{1}\right)-F^{t, s}\left(x^{0}, x^{1}\right)} & t \tau \\
= & \frac{s}{t} \frac{F^{t, s}\left(x^{0}, x^{1}+s \tau\right)-F^{t, s}\left(x^{0}, x^{1}\right)}{s \tau} \\
& +\frac{F^{t, s}\left(x^{0}+t \tau, x^{1}+s \tau\right)-F^{t, s}\left(x^{0}, x^{1}+s \tau\right)}{t \tau}-\frac{F^{t, s}\left(x^{0}+t \tau, x^{1}\right)-F^{t, s}\left(x^{0}, x^{1}\right)}{t \tau} \\
& +\frac{A}{t}\left[F^{-t, s}\left(x^{0}+t \tau, x^{1}+s \tau\right)+F^{t, s}\left(x^{0}+t \tau, x^{1}+s \tau\right)\right] \\
& +\frac{B}{t}\left[F^{-t,-s}\left(x^{0}+t \tau, x^{1}+s \tau\right)-F^{t, s}\left(x^{0}+t \tau, x^{1}+s \tau\right)\right] \\
& +\frac{C}{t}\left[F^{t,-s}\left(x^{0}+t \tau, x^{1}+s \tau\right)+F^{t, s}\left(x^{0}+t \tau, x^{1}+s \tau\right)\right] .
\end{aligned}
$$

In the limit $\tau \rightarrow 0$, when our lattice tends to recover all the space-time, we get the following set of four partial differential equations (one for each possible value of the couple $t, s$ of the first jump):

$$
-\frac{\partial F^{t, s}}{\partial x^{0}}=\frac{s}{t} \frac{\partial F^{t, s}}{\partial x^{1}}+\frac{A}{t}\left(F^{-t, s}+F^{t, s}\right)+\frac{B}{t}\left(F^{-t,-s}-F^{t, s}\right)+\frac{C}{t}\left(F^{t,-s}+F^{t, s}\right)
$$

where we neglected the arguments $\left(x^{0}, x^{1}\right)$ of the functions.

If we define now the following four linear combinations of the four functions $F^{t, s}$ :

$$
\begin{aligned}
& \phi=F^{1,1}+F^{-1,-1}+F^{1,-1}+F^{-1,1} \\
& \chi=F^{1,1}+F^{-1,-1}-F^{1,-1}-F^{-1,1} \\
& \psi=-F^{1,1}+F^{-1,-1}-F^{1,-1}+F^{-1,1} \\
& \omega=-F^{1,1}+F^{-1,-1}+F^{1,-1}-F^{-1,1}
\end{aligned}
$$

we can build a new equivalent set of equations by combining equations (4.6):

$$
\begin{aligned}
& \partial \phi / \partial x^{0}+\partial \chi / \partial x^{1}=2(C-B) \psi \\
& \partial \chi / \partial x^{0}+\partial \phi / \partial x^{1}=2(A-B) \omega \\
& \partial \psi / \partial x^{0}+\partial \omega / \partial x^{1}=2(A+C) \phi \\
& \partial \omega / \partial x^{0}+\partial \psi / \partial x^{1}=0 .
\end{aligned}
$$


By derivation and successive linear combination of equations (4.8) we have

$$
\begin{aligned}
\square \phi=2(C-B) \partial \psi / \partial x^{0}-2(A-B) \partial \omega / \partial x^{1} \\
=2(A-2 B+C) \partial \psi / \partial x^{0}-4(A-B)(A+C) \phi \\
\square \chi=2(A-B) \partial \omega / \partial x^{0}-2(C-B) \partial \psi / \partial x^{1}=2(A-2 B+C) \partial \omega / \partial x^{0} \\
\square \psi=2(A+C) \partial \phi / \partial x^{0}=-2(A+C) \partial \chi / \partial x^{1}+4(A+C)(C-B) \psi \\
\square \omega=-2(A+C) \partial \phi / \partial x^{1}=2(A+C) \partial \chi / \partial x^{0}-4(A+C)(A-B) \omega
\end{aligned}
$$

(where $\square$ is a two-dimensional d'Alembert operator) and if we pose

$$
B=\frac{1}{2}(A+C), \quad 2\left(A^{2}-C^{2}\right)=m^{2}
$$

we finally have

$$
\begin{aligned}
& \left(\square+m^{2}\right) \phi=0, \quad \square \chi=0 \\
& \left(\square+m^{2}\right) \psi=-2(A+C) \partial \psi / \partial x^{1} \\
& \left(\square+m^{2}\right) \omega=2(A+C) \partial \chi / \partial x^{0} .
\end{aligned}
$$

We now make the following three remarks.

(a) We can interpret the first equation of (4.11) as a Klein-Gordon equation. The function $\phi$, which satisfies this Klein-Gordon equation, is the complete average of a function $f$ over all the possible final points reached following all the possible trajectories of infinite jumps: in fact in this function, as we can see from (4.7), we consider now also the first jump by supposing that the four possibilities for the signs of $t, s$ are equiprobables.

(b) The functions $\chi, \psi, \omega$ which satisfy the remaining equations in (4.11) are not averages like $\phi$ and seem to us to constitute only a formal tool in the deduction of the equation for the complete average $\phi$. However, we see that in (4.11) the equation for $\phi$ is not coupled at all with the other equations for $\chi, \psi, \omega$ so that the solution of the Klein-Gordon equation is absolutely independent from the solutions of the rest of the system.

(c) The previous derivation of (4.11) from (4.8) shows that each solution $(\phi, \chi, \psi, \omega)$ of $(4.8)$ is a solution of $(4.11)$, but it is possible to show that not all the solutions of (4.11) are solutions of (4.8). Indeed, for example, we can verify by direct calculation that

$$
\phi=\exp (\mathrm{ip} \cdot x) \quad\left(\text { with } p^{2}=m^{2}\right), \quad \chi=\psi=\omega=0
$$

is a solution of (4.11) but it is not a solution of (4.8). Therefore it is important to analyse the following question: we proved the statement 'the function $\phi$ defined as a stochastic average in (4.7) and satisfying the system (4.8) always is a solution of a Klein-Gordon equation'; what about the inverse statement 'all the solutions of a Klein-Gordon equation are interpretable as stochastic averages satisfying a system like (4.8)'? We will show here that this inverse statement also holds in the following sense: if $\phi$ is an arbitrary solution of the Klein-Gordon equation we can always determine the functions $\chi, \psi, \omega$ in such a way that $(\phi, \chi, \psi, \omega)$ is a solution of (4.8). In fact, if $\phi$ is an arbitrary solution of the Klein-Gordon equation in (4.11), we can 
choose $\chi$ as an arbitrary solution of $\square \chi=0$ and then we determine $\psi$ and $\omega$ as follows

$$
\psi=\frac{1}{C-A}\left(\frac{\partial \phi}{\partial x^{0}}+\frac{\partial \chi}{\partial x^{1}}\right), \quad \omega=\frac{1}{A-C}\left(\frac{\partial \chi}{\partial x^{0}}+\frac{\partial \phi}{\partial x^{1}}\right) .
$$

It is only a matter of calculation to show that our $(\phi, \chi, \psi, \omega)$ is now a solution of $(4.8)$ (with $B=\frac{1}{2}(A+C)$ ) and of (4.11).

\section{Conclusion}

Despite the fact that the introduction of a square space-time is fundamentally noncovariant we get, at the end of the limit process $\tau \rightarrow 0$, a field equation which is covariant. That situation becomes clear if we think that a Markov process happens on the space of the possible velocities of the particle: it is evident from (3.2) that the set of these velocities is the set of the four possible space-time directions of the velocity of light. In that sense the ensemble of events of our Markov process is covariant exactly like the final wave equation, even if the space-time trajectories are, of course, non-covariant. Moreover, we point out that the non-covariance of the space-time lattice disappears when $\tau \rightarrow 0$ in the sense that in this limit our points recover all the space-time positions and the non-covariant parameter $\tau$ doesn't play any role in the 'continuous' final theory. In a word: the lattice of the 'possible' positions of a particle really doesn't exist; only the real trajectories exist and are constituted by choices between possible events in the covariant velocity space.

Finally, we remark that the fact that we developed our construction on a twodimensional space-time can be considered as a real limitation. From that standpoint the authors intend to carry out, in successive papers, the analysis of the possibility of the application of this method to the four-dimensional space-time and to the twoparticle case in the configuration space, in order to generalise completely a line of stochastic demonstration of the quantum equations that seems to be very suggestive because of the clearness of their construction.

\section{References}

Aspect A 1975 Phys. Lett. 54 A 117

1976 Phys. Rev. 14 D 1944

Aspect A, Grangier P and Roger G 1981 Phys. Rev. Lett. 47460

- 1982 Phys. Rev. Lett. 4991

Avez A 1976 Interprétation probabiliste d'equations aux derivées partielles hyperboliques normales (Paris, preprint)

Bell J S 1964 Physics 1195

Blokhintsev D I 1974 Fiz. Elem. Chastits. At. Yad. 5606

Bohm D 1952 Phys. Rev. 85 166, 180

1953 Phys. Rev. 89458

Bohm D and Vigier J P 1954 Phys. Rev. 96208

- 1958 Phys. Rev. 1091882

Bruno M, D'Agostino M and Maroni C 1977 Nuovo Cimento 40 B 142

Cerofolini G F 1980 Nuovo Cimento $\mathbf{5 8}$ B 286

Clauser J F 1976 Phys. Rev. Lett. 361223

Cramer H 1955 The Elements of Probability Theory (New York: Wiley)

Cufaro Petroni N, Droz-Vincent Ph and Vigier J P 1981 Lett. Nuovo Cimento 31415 
Cufaro Petroni N and Vigier J P 1979a Phys. Lett. 73 A 289

- 1979b Lett. Nuovo Cimento 26149

- 1981 Phys. Lett. 81 A 12

1982 Phys. Lett. 88 A 272

1983 Phys. Lett. 93 A 383

Dirac P A M 1951 Nature 168906

Einstein A 1924 Schweiz. Nat. Gesell. Ver. 8585

Einstein A, Podolsky B and Rosen N 1935 Phys. Rev. 47777

Falciglia F, Iaci G and Rapisarda V A 1979 Lett. Nuovo Cimento 26327

Faraci G, Gutkowsky S, Notarrigo S and Pennisi A R 1974 Lett. Nuovo Cimento 9667

Feynman R P 1949 Phys. Rev. 76 749, 769

Frederick C 1976 Phys. Rev. 13 D 3183

Freedman S J and Clauser F 1972 Phys. Rev. Lett. 28938

Fry E S and Thompson R C 1976 Phys. Rev. Lett. 37465

Garuccio A, Rapisarda V A and Vigier J P 1981 Lett. Nuovo Cimento 32451

Garuccio A and Rapisarda V A 1981 Nuovo Cimento 65 A 269

Guerra F and Ruggiero P 1978 Lett. Nuovo Cimento 23529

Holt R A and Pipkin F M 1974 Harvard, preprint

Janossy L 1972 Found. Phys. 29

Kasday L, Ullman J and Wu C S 1975 Nuovo Cimento 25663

Lehr W and Park J 1977 J. Math. Phys, 181235

March A 1934 Z. Phys. 104 93, 161

- 1937 Z. Phys. 105620

Markov M A 1958 Hyperons and mesons (Moscow: Fizmatgiz)

Namsrai K 1980 Found. Phys. 10 353, 731

Nelson E 1966 Phys. Rev. 1501079

Vigier J P 1979 Lett. Nuovo Cimento 24 258, 265

1980 Lett. Nuovo Cimento 29467

Wilson A R, Lowe J and Butt D K 1976 J. Phys. G: Nucl. Phys. 2613

Yukawa H 1966 Res. Inst. Fund. Phys. (Kyoto University) PIEP-55 